\title{
PORTFOLIO SELECTION WITH UNCERTAINTY MEASURES CONSISTENT WITH ADDITIVE SHIFTS
}

\section{Rosella Giacometti, Sergio Ortobelli, Tomáš Tichý*}

\begin{abstract}
:
Assuming a non-satiable risk-averse investor, the standard approach to portfolio selection suggests discarding of all inefficient investment in terms of mean return and its standard deviation ratio within its first step. However, in literature we can find many alternative dispersion and risk measures that can help us to identify the most suitable investment opportunity. In this work two new dispersion measures, fulfilling the condition that "more is better than less" are proposed. Moreover, their distinct characteristics are analysed and empirically compared. In particular, starting from the definition of dispersion measures, we discuss the property of consistency with respect to additive shifts and we examine two dispersion measures that satisfy this property. Finally, we empirically compare the proposed dispersion measures with the standard deviation and the conditional value at risk on the US stock market. Moreover, within the empirical example the so called "alarm" is incorporated in order to predict potential fails of the market.
\end{abstract}

Keywords: alarm signal, dispersion measure, investment, Sharpe ratio, stochastic dominance, systemic risk

\section{JEL Classification: C58, G11}

\section{Introduction}

In the 1950s Markowitz and Tobin proposed the following selection rule for a non-satiable risk-averse investor: among a given set of investment alternatives, which includes the set of securities available at the market as well as all possible linear combinations of these basic

* Rosella Giacometti, Department MSIA, University of Bergamo, Via dei Caniana 2, 24127 Bergamo, Italy; Sergio Ortobelli Lozza, Department MSIA, University of Bergamo, Via dei Caniana 2, 24127 Bergamo, Italy and Department of Finance, Faculty of Economics, VŠB-Technical University of Ostrava, Sokolská 33, 70121 Ostrava, Czech Republic; Tomáš Tichý, Department of Finance, Faculty of Economics, VŠB-Technical University of Ostrava, Sokolská 33, 70121 Ostrava, Czech Republic (tomas.tichy@vsb.cz).

This paper has been supported by the Italian funds ex MURST 60\% 2013 and 2014 and MIUR PRIN MISURA Project, 2013-2015 (R. Giacometti and S. Ortobelli). The research was also supported through the Czech Science Foundation (GACR) under Project No. 13-13142S (T. Tichý) and No. 15-23699S (S. Ortobelli) and through SP2015/15, an SGS Research Project of VSB-TU Ostrava, and furthermore by the European Social Fund in the framework of CZ.1.07/2.3.00/20.0296. 
securities, the efficient set of alternatives is obtained by discarding the investments that exhibit lower mean and higher risk (variance) than any member of a given set. However, in order to discard the inefficient investment some measure of risk and/or dispersion must be adopted.

Recently, many dispersion and risk measures, as an alternative to the classic case of standard deviation (variance), have been proposed either for their theoretical characteristics or for their more practical appeal. In particular, we recall the recent classification of coherent measures of risk (Artzner et al., 2000), the mean-variance and mean-semivariance approaches (Markowitz, 1959), mean absolute deviation (MAD) and semi-MAD approaches (see for example Konno and Yamazaki, 1991; Speranza, 1993, Ogryzak and Ruszczynski, 1999 and 2001 or Zenios and Kang, 1993), the mean-dispersion approach in the Dybvig's distributional analysis of portfolio choice (Dybvig, 1988), and many others (see e. g. Rachev et al., 2008 and the references therein).

It is well known that Markowitz and Tobin's selection rule is optimal for all nonsatiable risk-averse investors under particular assumptions on return distribution or utility function but it fails as an optimal general decision rule. In particular, under the assumptions of "limited liability" and "no short sale", portfolios of gross returns are positive random variables and the mean-dispersion Markowitz and Tobin's rule is not optimal (Ortobelli et al., 2009 and Petronio et al., 2014).

This observation boosts the interest in the analysis of different dispersion measures defined for positive random variables, under some assumptions of adherence to the reality of the market. In particular, we are interested in dispersion measures that consider preferable random wealth $W$ plus a positive constant $a$ than the only random wealth $W$. On the other hand, it is well known that the classic dispersion measures, which are the deviation measures (Rockafeller, 2006) used in portfolio selection problems do not take into account that $W+a$, is preferred to $W$ by each non-satiable investor. Therefore the consistency with additive shift is alternative and it has the same motivation of translation invariance property of coherent risk measures (see Artzner et al., 2000).

Our aim in this paper is to investigate possible dispersion measures that satisfy such property and suggest alternative portfolio selection problems. We proceed as follows. We first study the properties of the dispersion measures that satisfy these criteria and we analyse and compare two new measures with the classic benchmark in literature, the standard deviation and the example of coherent risk measures, the conditional value at risk (CVaR). The first dispersion measure that satisfies the above properties - in other words that is suitable for positive and fat tailed random variables - is the covariance between the gross return $1+R$ and its logarithm, where $R$ is the rate of return of an admissible portfolio. This dispersion measure is consistent with risk-averse investors' preferences and it gives different values to random variables that differ by an additive constant. In addition, we empirically observe the convexity of this measure, which, however, we were not able to prove theoretically. Moreover, analogously to semivariance, we also introduce a measure that account for the downside risk.

Finally, we propose an empirical comparison among the standard deviation, conditional value at risk, and the new measures consistent with additive shifts. For this purpose we consider allocation problems where the investors maximize different performance ratios based on different concepts of risk. We determine the ex-post wealth obtained maximizing different mean/risk ratios. The portfolios obtained with this methodology represent 
the optimal investors' choices in the different approaches. Moreover, we analyse the portfolio diversification and turnover of these strategies.

The paper is organized as follows. In Section 2 we analyse the stochastic dominance characteristics of mean-dispersion approach and we introduce the properties of the two proposed dispersion measures. In Section 3 we compare the two dispersion measures with the standard deviation and the last section briefly concludes the paper.

\section{The Portfolio Allocation Problem with Dispersion Measures Consistent with Additive Shifts}

In this section we study admissible dispersion measures for the portfolio selection problem analysing their consistency with the maximization of the expected utility. In particular, we consider the portfolios gross returns, $x^{\prime} z$, positive random variables defined on the probability space $(\Omega, \mathfrak{I}, P)$, where $x \in R_{+}^{n}$ is the vector of non-negative allocations among $n$ risky limited liability investments with gross returns ${ }^{1} z=\left[z_{1}, \ldots, z_{n}\right]^{\prime}$; moreover, $z_{0}$ denotes the riskless gross return. We assume that short sale is not allowed (i.e. $x_{i} \geq 0 ; \forall i$ ).

During the last years, we could observe a heated debate on the risk measures. In particular, Artzner et al. (2000) have provided a definition of coherent risk measures. A coherent risk measure applied to the portfolio selection problem with institutional restrictions is a non-positive real mapping $\rho$ that associates a real number $\rho\left(x^{\prime} z\right) \leq 0$, to each portfolio $x^{\prime} z{ }^{2}$ Such mapping has to satisfy the following axioms, in order to be a coherent risk measure:

1. For all portfolios $x^{\prime} z$ and all real numbers $a$ we have $\rho\left(x^{\prime} z+a z_{0}\right)=\rho\left(x^{\prime} z\right)-a$.

2. For all portfolios $x^{\prime} z$ and all $a \geq 0$ we have $\rho\left(a x^{\prime} z\right)=a \rho\left(x^{\prime} z\right)$.

3. For any $x^{\prime} z$ and $y^{\prime} z \rho\left(x^{\prime} z+y^{\prime} z\right) \leq \rho\left(x^{\prime} z\right)+\rho\left(y^{\prime} z\right)$.,

4. For any $x^{\prime} z \leq y^{\prime} z, \rho\left(x^{\prime} z\right) \leq \rho\left(y^{\prime} z\right)$

Under these assumptions a typical coherent risk measure associated to the portfolio selection problem is $\bar{\rho}\left(x^{\prime} z\right)=-\frac{E\left(x^{\prime} z\right)}{z_{0}}$. As argued by Rachev et al. (2008), part of the properties of a coherent risk measure can be subject to many criticisms.

On axiom 1 we can note that even if it points out the difference on the preferences due to an additive shift, the property appears too restrictive and it can be relaxed.

Axiom 4 expresses a risk preference order for ordered random variables. However, we need additional considerations to display a preference order when there exists a stochastic

1 We define the $i$-th gross return between time $t$ and time $t+1$ as $z_{i, t} z_{i, t}=\frac{P_{t+1, i}+d_{[t, t+1], i}}{P_{t, i}}$, where $P_{t, i}$ is the price of the $i$-th asset at time $t$ and $d_{[t, t+1], i}$ is the total amount of cash generated by the instrument between $t$ and $t+1$.

2 A coherent risk measure is negative applied to positive random variables (as the portfolio of gross returns) but it is not negative for all random variables (see Artzner et al. 2000). 
dominance ordering ${ }^{3}$ between the portfolios. That is, if $\rho(X) \leq \rho(Y)$, we generally do not know if $X$ is preferable to $Y$ by a non-satiable or risk averse investors and conversely we do not know which relationship exists between a coherent risk measure associated to $X$ and $Y$, when $X$ is preferred to $Y$ by some class of investors.

As an example, the variance does not satisfy any of the four axioms. In particular, we can discuss axiom 3. The variance of the sum of two positively correlated portfolios is greater than the sum of the variances. In this sense variance is a dispersion measure more risk preserving than a coherent measure of risk. Conversely, when the random portfolios are negatively correlated, variance verifies axiom 3 and it favours the diversification of risk. However, while variance does not verify the above axioms, standard deviation verifies axioms 2 and 3 (but not axioms 1 and 4).

This observation indicates that there could exist a dispersion measure, or increasing function of dispersion measures, which verify other not less interesting properties even if they do not support the four axioms of coherence. On the other hand, several dispersion measures and risk measures are still widely used in portfolio allocation problems (see Rachev et al. (2008)). Recall that a dispersion measure is any law invariant measure $\sigma$ (i.e., if $X$ and $Y$ have the same distributions, then $\sigma_{X}=\sigma_{Y}$ that satisfies the following characteristics:

1. (positive homogeneous) $\sigma_{\beta X}=\beta \sigma_{X}$ for every positive real $\beta \geq 0$;

2. (positive) $\sigma_{X} \geq 0$ and $\sigma_{X}=0$ if and only if is a constant a.s.;

3. (consistent with additive shift) $\sigma_{X+t} \leq \sigma_{X}$ for every real positive $t \geq 0$.

Most of the dispersion measures used in financial modeling are deviation measures. Therefore, by definition (see Rockafeller et al., 2006) all of them are the subadditive (i.e., $\sigma_{X+Y} \leq \sigma_{X}+\sigma_{Y}$ for any random variable $X$ and $Y$ ) dispersion measures for which property 3 is satisfied as equality (i.e., $\sigma_{X+t}=\sigma_{X}$ for any real $t$ ). Differently from coherent risk measures dispersion measures cannot be consistent with first or second stochastic dominance order. Even for this reason dispersion measures must be used in a different way with respect to coherent risk measures (see Ortobelli et al., 2005) and also Kopa and Tichý, 2014). Although most of the dispersion measures are consistent with risk averse

3 Recall that a portfolio $x^{\prime} z$ stochastically dominates in the first order sense (FSD) a portfolio $y^{\prime} z$ if and only if for every increasing utility function $u$ it holds that $\mathrm{E}\left[u\left(x^{\prime} z\right)\right] \geq \mathrm{E}\left[u\left(y^{\prime} z\right)\right]$, while the inequality strictly holds for at least one $u$. Equivalently, $x^{\prime} z$ dominates $y^{\prime} z$ in the FSD sense if and only if $P\left(x^{\prime} z \leq t\right) \leq P\left(y^{\prime} z \leq t\right)$ for any real $t$ and strictly for some $t$. Analogously, we say that a portfolio $x^{\prime} z$ stochastically dominates in the second order sense (SSD) a portfolio $x^{\prime} z$ if and only if for every increasing, concave utility function $u$ it holds that $\mathrm{E}\left[u\left(x^{\prime} z\right)\right] \geq \mathrm{E}\left[u\left(y^{\prime} z\right)\right]$, while the inequality must strictly hold for at least one $u$. Equivalently, $x^{\prime} z$ dominates $y^{\prime} z$ in the SSD sense if and only if $\int_{-\infty}^{t} F_{x^{\prime} z}(v) d v \leq \int_{-\infty}^{t} F_{y^{\prime} z}(v) d v$ for every real $t$ and strictly for some $t$ (see, among others, Hanoch and Levy (1969) or Hadar and Russel (1969)). We also say that a portfolio $x^{\prime} z$ stochastically dominates in the Rothschild-Stiglitz sense (R-S) a portfolio $y^{\prime} z$ if and only if for every concave utility functions $u$ it holds that $\mathrm{E}\left[u\left(x^{\prime} z\right)\right] \geq \mathrm{E}\left[u\left(y^{\prime} z\right)\right]$, while the inequality is strict for at least one $u$. Equivalently, $x^{\prime} z$ dominates $y^{\prime} z$ in the R-S sense if and only if $\mathrm{E}\left[x^{\prime} z\right] \geq \mathrm{E}\left[y^{\prime} z\right]$ and simulatenously $x^{\prime} z$ dominates $y^{\prime} z$ in the SSD sense (see Rothschild and Stiglitz (1970)). Note, however, that in economics and finance one can use many other stochastic orders - see, among others, Levy (1992), Shaked and Shanthikumar (1993) or Ortobelli et al. (2009). 
investors' preferences (see Bauerle and Müller, 2006) only few dispersion measures are strictly consistent with additive shifts, i.e., $\sigma_{X+t}<\sigma_{X}$ for every $\mathrm{t}>0$. Generally, consistency with respect to additive shift considers and valorises preferences of non-satiable investors due to a positive additive shift. In the following subsection we introduce and analyse the properties of two dispersion measures consistent with additive shifts.

\subsection{Dispersion measures consistent with additive shifts}

Assume any portfolio $x^{\prime} z$ belonging to $L^{q}=\left\{X \mid \mathrm{E}\left(|X|^{q}\right)<\infty\right\}$ for some real $q$ greater than or equal to one. Thus, if there exists finite $\mathrm{E}\left(\log \left(x^{\prime} z\right)\right)$, we can consider

$$
\operatorname{cov}\left(x^{\prime} z, \log x^{\prime} z\right)=\mathrm{E}\left(x^{\prime} z \log x^{\prime} z\right)-\mathrm{E}\left(x^{\prime} z\right) \mathrm{E}\left(\log x^{\prime} z\right)
$$

as dispersion measure because $\operatorname{cov}\left(x^{\prime} z, \log x^{\prime} z\right)<\infty .{ }^{4}$ We denote with $\operatorname{colog}(X)$ the cov $(X, \log X)$ and in the following lemma we prove that colog can be used as dispersion measure.

Proposition 1. Assume that for each portfolio $x^{\prime} z$ there exists finite $\mathrm{E}\left(\log x^{\prime} z\right)$ and for some real $q$ greater than or equal to one $x^{\prime} z \in L^{q}$. Then $\operatorname{colog}\left(x^{\prime} z\right)$ is a dispersion measure, according to the above definition. In particular, for every non constant positive random variable $X$ and for each $t>0, \operatorname{colog}(X+t)<\operatorname{colog}(X)$ (i.e., it is strictly consistent with additive shifts).

Proof. For every $\left.\beta>0, \operatorname{cov}\left(\beta x^{\prime} z, \log \beta x^{\prime} z\right)\right)=\beta \operatorname{cov}\left(x^{\prime} z, \log x^{\prime} z\right)$. Thus, property 1 of dispersion measures holds. Let us now consider property 2 . For every $t>0$, the function $f(t)=t \log t$ is convex and the functions $g(t)=\log t$, and $h(t)=t^{m}$, with $m \in(0,1)$, are concave. Then, using Jensen inequality it follows, that:

$$
m \mathrm{E}\left(x^{\prime} z \log x^{\prime} z\right) \geq \mathrm{E}\left(x^{\prime} z\right) \log \left(\mathrm{E}\left(x^{\prime} z\right)\right)^{m} \geq \mathrm{E}\left(x^{\prime} z\right) \log \mathrm{E}\left[\left(x^{\prime} z\right)^{m}\right] \geq m \mathrm{E}\left(x^{\prime} z\right) \mathrm{E}\left[\log \left(x^{\prime} z\right)\right]
$$

Thus $\operatorname{cov}\left(x^{\prime} z \log x^{\prime} z\right) \geq 0$. Moreover, if $x^{\prime} z$ is constant, then $\operatorname{cov}\left(x^{\prime} z \log x^{\prime} z\right)=0$. Conversely, if $\operatorname{cov}\left(x^{\prime} z \log x^{\prime} z\right)=0$, we derive for every $m \in(0,1)$ that $\left(\mathrm{E}\left(x^{\prime} z\right)\right)^{m}=\mathrm{E}\left(\left(x^{\prime} z\right)^{m}\right)$. Therefore $x^{\prime} z=\mathrm{E}\left(x^{\prime} z\right)$ a.s. Finally we have to prove that $\mathrm{E}(X) \mathrm{E}[\log (X+t)-\log X]>\mathrm{E}[X(\log (X+t)-\log X]$. We can observe that for every $t>0$ and $x>0$ function $f(x)=x \log \left(1+\frac{t}{x}\right)$ is strictly concave and function $g(x)=\log \left(1+\frac{t}{x}\right)$ is strictly convex. Then, using Jensen inequality we obtain $\mathrm{E}(X) \mathrm{E}(\log (X+t)-\log X)>\mathrm{E}(X)\left(\log \left(1+\frac{t}{\mathrm{E}(X)}\right)\right)>\mathrm{E}[X(\log (X+t)-\log X)]$, and the thesis holds.

4 Let $g(r)=E\left(X^{r}\right)$ be the moments curve of a given positive random variable $X$. The moments curve $g(r)$ is an analytical function. Thus, for every positive random variable $X \in L^{q}$ with $q>1$ the moments curve $g(r)$ is defined on the interval $(0, q)$ and it follows that $\mathrm{E}(X \log X)=\left.\frac{\partial g(r)}{\partial r}\right|_{r=1}<\infty$. 
Although in this work we were not able to prove theoretically the convexity of colog, several empirical examples show that colog applied to gross returns is a convex measure, i.e., $\operatorname{colog}(a X+(1-a) Y) \leq a \operatorname{colog}(X)+(1-a) \operatorname{colog}(Y)$ for every $a \in[0,1]$ and for all positive random variables $X$ and $Y$. In order to illustrate the empirical convexity of colog we consider 2000 historical observations of three gross returns (3M, Mcdonald, Intel - stocks, which are components of DJIA index) and plot the values of colog by varying the portfolio composition in the 3-dimensional simplex: $S=\left\{\left(x_{1}, x_{2}, x_{3}\right) \mid x_{1}+x_{2}+x_{3}=1, x_{1}, x_{2}, x_{3} \geq 0\right\}$. Figure 1 shows the convex surface we get varying the portfolio composition of three assets. Any convex measure, that is positive homogeneous is also sub-additivite, since $\sigma_{X+Y} \leq a \sigma_{X / a}+(1-a) \sigma_{Y /(1-a)}=\sigma_{X}+\sigma_{Y}$. Therefore we believe that colog is a convex, subadditive dispersion measure.

In several cases investors optimize their choices using portfolios of log returns. The classic distributional assumption that justifies the mean variance approach consists in assuming a jointly Gaussian distribution for the vector of log returns $r=\left[r_{1}, \ldots, r_{n}\right]^{\prime}$ (with $r_{i}=\log \left(z_{i}\right)$ ). Under this distributional assumption the unitary future wealth (portfolio of gross returns) is approximated with the exponential of portfolio of log returns ${ }^{5}$ i.e., $x^{\prime} z \approx \exp \left(x^{\prime} r\right)$ and colog is given by $\operatorname{colog}\left(\exp \left(x^{\prime} r\right)\right)=\sigma_{x^{\prime} r}^{2} \exp \left(\mu_{x^{\prime} r}+\frac{\sigma_{x^{\prime} r}^{2}}{2}\right)$. Moreover, differently to variance, colog exists even when finite variance does not exist. ${ }^{6}$ Therefore according to many empirical and theoretical analyses on the return distributions (see Rachev and Mittnik (2000) and the references therein), colog can be used for heavy tailed portfolio distributions. The following proposition illustrates the most important property of colog in portfolio problems.

\section{Figure 1}

\section{Values of Portfolio Colog Obtained Varying the Portfolio of Composition among Three Assets}

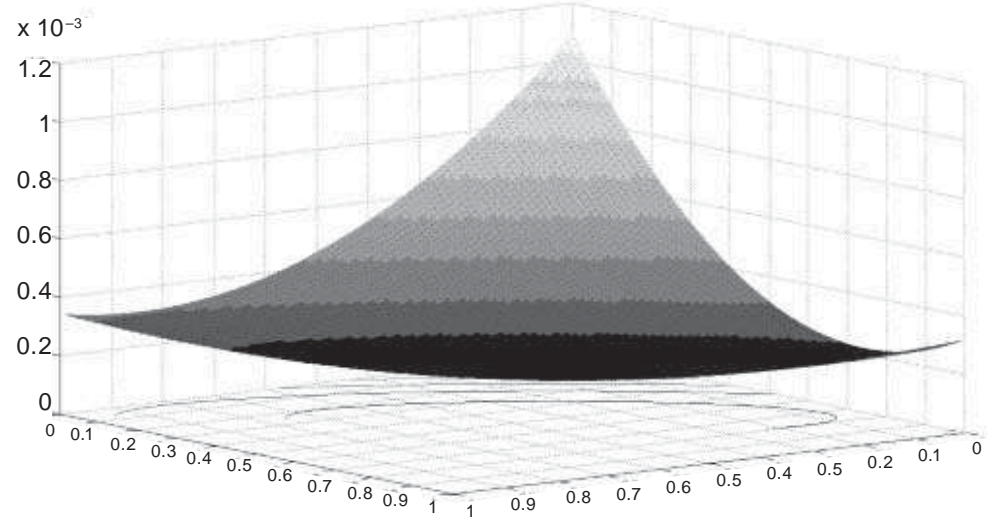

5 The portfolio of gross return (that is the unitary future wealth) is usually approximated with the exponential of portoflio of log returns. This approximation is good enough when daily or weekly $\log$ returns are used, but it is not advisable when monthly or yearly returns are used.

6 Generally, heavy tails of asset returns are modeled assuming stochastic models with infinite variance. In these cases, the following inequality holds $1<q<2$ where $\left.q=\max \left\{r>0 \mid \forall i=1, \ldots, n ; \mathrm{E}\left(\left(\tilde{z}_{i}\right)^{r}\right)<\infty\right\}\right\}$. 
Proposition 2. For every couple of positive non constant random variables and $Y$, if every risk-averse investor prefers $X$ to $Y$, then $\operatorname{colog}(Y) \geq \operatorname{colog}(X)$.

Proof. Assume that each risk averse investor prefer $X$ to $Y$, then for every concave utility function $u, E[u(X)] \geq E[u(Y)]$. For every $x>0$ the function $h(x)=-x \log x$ is concave, then

$$
-\mathrm{E}[X \log (X)] \geq-\mathrm{E}[Y \log (Y)]
$$

Thus, $\mathrm{E}[Y \log (Y)] \geq \mathrm{E}[X \log (X)]$. Moreover, from Lemma 1 it follows that $\mathrm{E}[X \log (X)] \geq \mathrm{E}[X] \mathrm{E}[\log (X)]$ and since the logarithmic function is concave $\mathrm{E}(X) \mathrm{E}(\log X) \geq \mathrm{E}(Y) \mathrm{E}(\log Y)$. Therefore, we proved $\mathrm{E}(Y \log (Y)) \geq \mathrm{E}(X \log (X)) \geq \mathrm{E}(X) \mathrm{E}(\log X) \geq \mathrm{E}(Y) \mathrm{E}(\log Y)$. And thus $\operatorname{colog}(Y) \geq \operatorname{colog}(X)$.

Hence, when the distribution functions of random portfolios are identified by the mean and the colog, the risk-averse investors will choose optimal portfolios between the solutions of the following constrained problem:

$$
\begin{gathered}
\min _{x} \operatorname{colog}\left(x^{\prime} z\right) \\
\text { s.t. } \\
x^{\prime} e=1 ; \quad x^{\prime} \mu=m \\
x_{i} \geq 0 ; i=1, \ldots, n
\end{gathered}
$$

for some parameters $m$, where $\mu=E(z)$ and $e=[1, \ldots, 1]^{\prime}$. Recall, that Markowitz (1959) recommends the use of semi-variance rather than variance in order to consider the downside risk. Analogously to semi-variance we can consider the following dispersion measure

$$
\operatorname{colog}\left(\min \left(x^{\prime} z, \mathrm{E}\left(x^{\prime} z\right)\right)\right)
$$

associated to the portfolio of gross returns $x^{\prime} z$. We denote with $\operatorname{cologdsr}\left(x^{\prime} z\right)$ the $\operatorname{colog}\left(\min \left(x^{\prime} z, \mathrm{E}\left(x^{\prime} z\right)\right)\right)$. Similarly to colog, also cologdsr is a dispersion measure consistent with additive shift as proved in the following corollary.

Corollary. Assume that for every portfolio $x^{\prime} z$ there exists finite $\mathrm{E}\left(\log x^{\prime} z\right)$. Then for some real $q$ greater than or equal to one we get that $x^{\prime} z \in L^{q}$. It follows that $\operatorname{cologdsr}\left(x^{\prime} z\right)$ is an admissible dispersion measure consistent with additive shifts.

Proof. Let us consider $h(X)=\min (X, \mathrm{E}(x))$ for any random variable $X$. Then for every positive non constant random variable $X$ and for every $t>0$,

$$
\operatorname{colog} \operatorname{dsr}(X+t)=\operatorname{colog}(h(X+t))<\operatorname{colog}(h(X))=\operatorname{colog} \operatorname{dsr}(X) .
$$

Clearly the above dispersion measures can be used to identify superior, ordinary, and inferior performance in a mean-risk framework. In this context the solution of the optimization problem:

$$
\begin{aligned}
& \max _{x} \frac{\mathrm{E}\left(x^{\prime} z\right)-z_{0}}{\sigma_{x^{\prime} z}} \\
& \sum_{i=1}^{n} x_{i}=1 ; x_{i} \geq 0
\end{aligned}
$$


is a portfolio that provides maximum reward per unit of risk $\sigma_{x^{\prime} z}$. Such a portfolio is generally called the market portfolio. The ratio between the expected excess returns $\mathrm{E}\left(x^{\prime} z\right)-z_{0}$ and the risk $\sigma_{x^{\prime} z}$ (that is colog of cologdsr of $x^{\prime} z$ ) is an extended Sharpe measure that is an alternative portfolio performance to the classic Sharpe ratio. For example, if we consider the presence of the riskless gross return $z_{0}$ we know that $\operatorname{colog}\left((1-\lambda) x^{\prime} z+\lambda z_{0}\right)<\operatorname{colog}\left((1-\lambda) x^{\prime} z\right)$ for any $\lambda \in(0,1)$. Thus, the ratio between the expected excess return $(1-\lambda)\left(x^{\prime} z-z_{0}\right)$ and the colog of the portfolio $(1-\lambda) x^{\prime} z+\lambda z_{0}$ is not constant (as for the Sharpe ratio) varying the parameter $\lambda$. In particular, we get

$$
\lim _{\lambda \rightarrow 1} \frac{(1-\lambda) \mathrm{E}\left(x^{\prime} z-z_{0}\right)}{\operatorname{colog}\left((1-\lambda) x^{\prime} z+\lambda z_{0}\right)}=\operatorname{sign}\left(\mathrm{E}\left(x^{\prime} z-z_{0}\right)\right) \infty .
$$

Moreover, we also observe that

$$
\lim _{\lambda \rightarrow 1} \frac{\partial \operatorname{colog}\left((1-\lambda) x^{\prime} z+\lambda z_{0}\right)}{\partial \lambda}=0
$$

and

$$
\lim _{\lambda \rightarrow 1} \frac{\partial^{2} \operatorname{colog}\left((1-\lambda) x^{\prime} z+\lambda z_{0}\right)}{\partial^{2} \lambda}=\frac{\operatorname{var}\left(x^{\prime} z\right)}{2 z_{0}}
$$

Therefore, if two fund separation theorem holds and all optimal portfolios can be seen as linear combination of the riskless and a market portfolio, this market portfolio should maximize the expected excess returns minimizing the convexity of the colog function on the line $(1-\lambda) x^{\prime} z+\lambda z_{0}$. Thus, when two fund separation holds under classic restrictions of the market (no short sales and limited liability), we deduce that a good approximation of the market portfolio is given by the portfolio that maximizes the ratio $\frac{\mathrm{E}\left(x^{\prime} z\right)-z_{0}}{\operatorname{var}\left(x^{\prime} z\right)}$ that
is also the optimal leverage ratio (see Peters, 2011).

\section{An ex-post Comparison among Risk Measures Evaluating the Presence of the Systemic Risk}

In this section we propose an ex-post comparison among portfolio models based on three different dispersion measures (namely standard deviation, colog and cologdsr) and the conditional value at risk (CVaR), defined as:

$$
\operatorname{CVaR}_{\alpha}(X)=\frac{-1}{\alpha} \int_{0}^{\alpha} F_{X}^{-1}(u) d u
$$

where $F_{X}^{-1}(p)=\inf \left\{x: \operatorname{Pr}(X \leq x)=F_{X}(x) \geq p\right\}$ is the $p$-quantile of $X$ the and it is the left inverse of the cumulative distribution function $F_{X}$. The conditional value at risk is not a dispersion measure and it cannot be used as a dispersion measure (see Ortobelli et al., 2005 and Rachev et al., 2008 and the reference therein). However, CVaR is a coherent risk measure consistent with second stochastic dominance order (choices of non satiable 
and risk averse investors), ${ }^{7}$ which has been often taken as benchmark among coherent risk measures. In particular, we consider as parameter $\alpha=0.05$ and we compare the ex-post performance of the four strategies on the US stock market (NASDAQ, NYSE and AMEX) during the last "decade" (January, 2000 to September, 2011). ${ }^{8}$ In order to guarantee a good liquidity of the traded assets we consider only those US stocks for which the daily average of traded value is higher than 100 million dollars. We can get it as follows:

\section{Daily average of traded value $=$ Closing price $\times$ Daily volume,}

and the average is computed over the last six months (125 working days). These stocks are preselected among all stocks traded in the US stock market, which have been active during the last six months. Moreover, since we value the ex-post value of wealth of four models during a period with high volatility of the markets and presence of systemic risk, we introduce an "alarm" that tells us about the presence of systemic risk. The "alarm" is a simple rule that counts the assets whose average returns on the last 10 days is lower than the mean on the last 100 working days. If the number of these assets is higher than $90 \%$ of the assets we deduce that systemic risk is probably present on the market since $90 \%$ of the assets are losing the value. Therefore, in presence of systemic risk we do not invest in the market for any strategy. When we do not observe the presence of systemic risk, we suggest investing to the 100 US stocks with the highest Sharpe ratio.

In particular, starting from January 1, 2000 we preselect the 100 stocks with the highest performance ratio $\frac{\mathrm{E}\left(x^{\prime} z-1\right)}{\sigma_{x^{\prime} z}}$ and do the same every 20 working days during the last "decade" (January, 2000 to September, 2011) if the proposed alarm does not find out the presence of systemic risk. The functional $\sigma_{x^{\prime} z}$ is either the standard deviation, or the colog or the cologdsr or the CVaR. In the performance ratio we implicitly assume that the riskless investment is not allowed and thus its gross return $z_{0}$ is equal to 1 . In order to find the right trade-off between a statistical approximation of the historical series and the number of historical observations, we have to reduce the dimensionality of the portfolio problem. Thus, as suggested by Ortobelli and Tichy (2011), we perform a PCA of the returns of the stocks used in order to identify the few factors $f_{j}$ with the highest return variability. We apply the PCA on the Pearson correlation matrix and then we further reduce the variability of the error by regressing the series on these factors $f_{j}$ so that we approximate the returns:

$$
z_{i}=b_{i, 0}+\sum_{j=1}^{s} b_{i, j} f_{j}+\varepsilon_{i}
$$

On the preselected approximated gross returns (that presents a good liquidity) we maximize the mean-risk ratio $\frac{\mathrm{E}\left(x^{\prime} z-1\right)}{\sigma_{x^{\prime} z}}$, such that the vector of weights $x$ belongs to:

$$
S=\left\{x \in R^{n} \mid \sum_{i=1}^{n} x_{i}=1 ; x_{i} \geq 0 ; x_{i} \leq 0.1\right\}
$$

7 For more details about measures consistent with second stochastic dominance ordering see, among others, Ogryczak and Ruszczyński $(1999,2002)$.

8 We take the dataset from DataStream. We choose the US stock market evolution due to its high liquidity and in order to capture both market downfalls in recent years. 
that is, a short sale is not allowed and we cannot invest more than $10 \%$ in a single asset. Therefore we can compare the ex-post wealth sample paths obtained using the different optimal portfolios where the risk $\sigma_{x^{\prime} z}$ is optimized assuming either the standard deviation, or the colog or the cologdsr or the CVaR.

We use a moving window of 125 working days (about 6 months) for the computation of each optimal portfolio and we recalibrate the portfolio every month. Then, considering an initial wealth $W_{t_{0}}=1$ that we invested on January 1,2000, we evaluate the ex-post wealth sample paths for the four strategies. Thus, at the $k$-th optimization $(k=0,1,2, \ldots, m)$, four main steps are performed to compute the ex-post final wealth.

Step 1 Evaluate the presence of systemic risk. In presence of systemic risk the ex-post final wealth is constant during the period $\left[t_{k}, t_{k+1}\right]$ (where $\left.t_{k+1}=t_{k}+20\right)$, i.e.:

$$
W_{t_{k+1}}=W_{t_{k}} .
$$

In such case we repeat Step 1, otherwise we continue to Step 2.

Step 2 Preselect the 100 (liquid) stocks with the highest performance ratio $\frac{\mathrm{E}\left(x^{\prime} z-1\right)}{\sigma_{x^{\prime} z}}$, while the gross returns of these assets are approximated applying a PCA on the original series.

Step 3 Determine the market portfolio $x_{M}^{(k)}$ that optimizes the portfolio problem (3) with the further constraint $x_{i} \leq 0.1$.

Step 4 Since we recalibrate the portfolio every 20 days, the ex-post final wealth after each round is given as:

$$
W_{t_{k+1}}=W_{t_{k}}\left(\left(x_{M}^{(k)}\right)^{\prime} z_{\left(t_{k+1}\right)}^{(e x p o s t)}\right)
$$

where $z_{\left({ }_{k+1}\right)}^{(\text {expost })}$ is the vector of observed gross returns in the period $\left[t_{k}, t_{k+1}\right]$ (where $\left.t_{k+1}=t_{k}+20\right)$.

All these steps are repeated until the observations are available.

\subsection{Empirical results}

The results of this analysis are reported in Figures 2 and 3. In Figure 2 we report the ex-post comparison of the sample paths of wealth obtained with the different portfolio strategies. Figure 2 shows the following.

- Although all strategies based on a different concept of risk present higher final wealth than the classic one based on the maximization of the Sharpe ratio, the strategies with the best ex-post performance are clearly those based on the maximization of $\frac{\mathrm{E}\left(x^{\prime} z-1\right)}{\operatorname{cologdsr}\left(x^{\prime} z\right)}$ and $\frac{\mathrm{E}\left(x^{\prime} z-1\right)}{C V a R_{0.05}\left(x^{\prime} z\right)}$. On the one hand, the strategy based on the CVaR presents the highest ex-post final wealth and it appears the most conservative since it increases more during the last period of crisis. On the other hand, during some years the strategy based on cologdsr present better performance than the one based 
on CVaR and both strategies (CVaR and cologdsr) take into account the downside risk and present similar stochastic dominance properties.

- The alarm inserted to detect the presence of systemic risk works well, since it is able to identify and forecast the largest period of systemic risk of the recent crises (subprime crisis 2007-2009 and the country credit risk crisis 2010-2011).

The turnover and diversification behaviour do not change too much among the four strategies. Thus in Figure 3 we describe the portfolio turnover and its diversification for the Cologdsr strategy (that is similar for all the four strategies). In Figure 3 it is examined how the portfolio composition of this strategy changes during the ex-post period and in particular during the period of crisis 2008-2011. In the first sub-figure (Figure 3a) we point out the percentages $\varphi_{k}(k=1, \ldots, 145)$ of the portfolio changed every 20 days obtained by the formula:

$$
\phi_{k}=\sum_{i=1}^{n}\left|x_{M, i}^{(k)}-x_{M, i}^{(k-1)}\right|
$$

where $x_{M, i}^{(k)}$ is the $\mathrm{i}$-th component of the optimal portfolio $x_{M}^{(k)}$. In particular $\varphi_{k}$ should belong to the interval $[0,2]$, where the value 0 means that the portfolio composition is not changed during the period $\left[t_{k-1}, t_{k}\right]$ while the value 2 corresponds to the case we sell the portfolio and we buy a completely different portfolio. The last sub-figure (Figure 3(b)) shows how the portfolio changes its composition during the crisis 2008-2011. In particular Figure 3(b) points out the quantity of: (1) assets used (i.e. those assets whose percentages are greater than zero $\left.x_{M, i}^{(k)}>0, i=1, \ldots, n\right) ;(2)$ entering assets; (3) exiting assets.

\section{Figure 2}

Ex-post Comparison among Four Portfolio Strategies Based on Different Concept of Risk: Standard Deviation (Sharpe), Colog (colog), Cologdsr (cologdsr) and CVaR (CVaR)

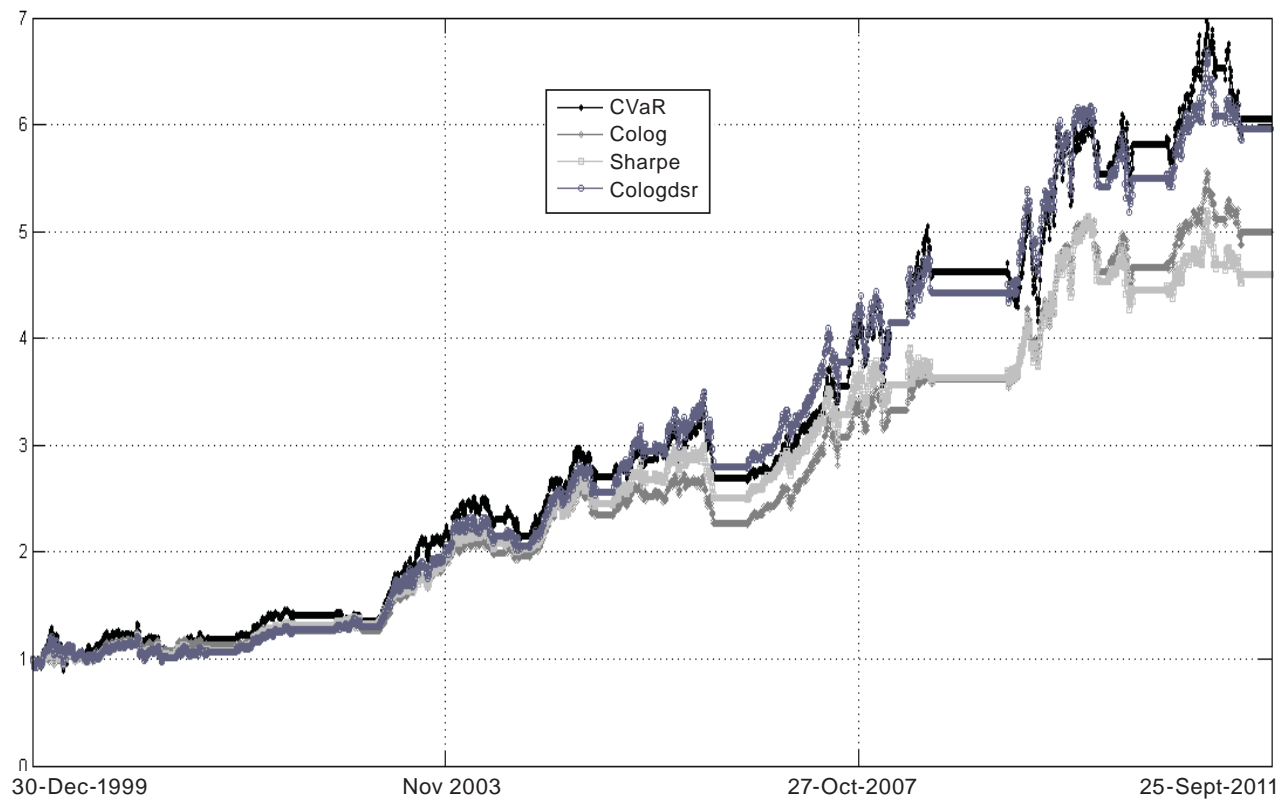




\section{Figure 3}

\section{Portfolio Turnover and Diversification of the Cologdsr Strategy}

a) Percentage of the monthly portfolio variations

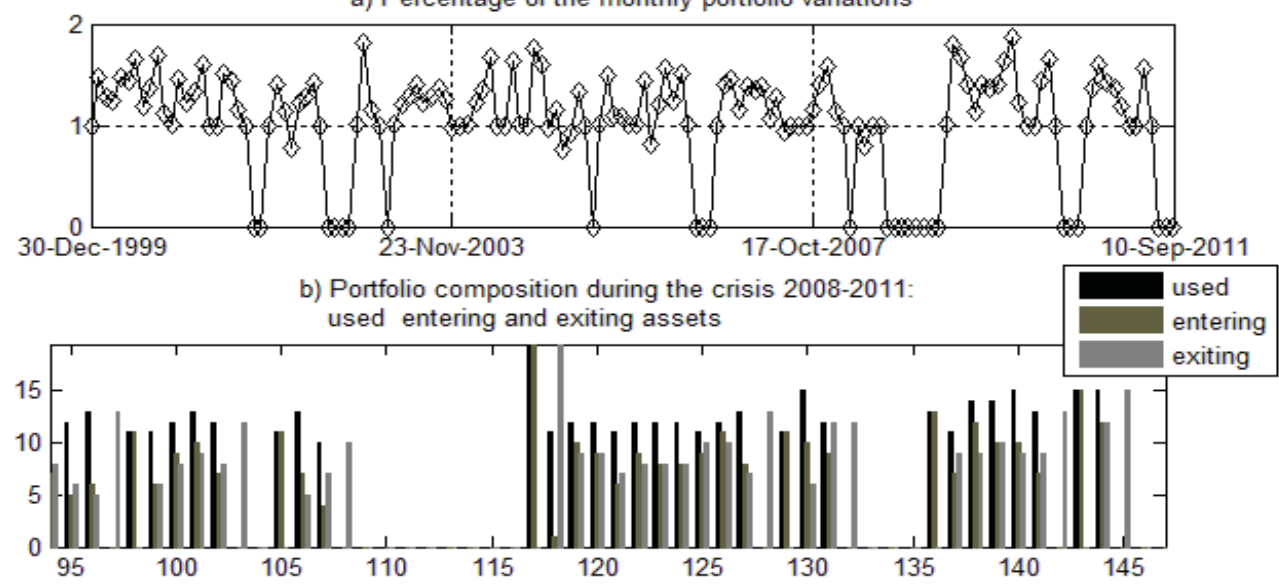

The portfolio is not strongly diversified among all preselected assets since there are always several assets in which the strategy suggests to invest the maximum amount (i.e. 10\%). The average of the number of assets in which the strategies suggest to invest in this decade is 15.213 for the colog strategy, 15.167 for the cologdsr strategy, 14.971 for the Sharpe strategy and 14.474 for the CVaR one. Moreover, we also observe that there is a strong turnover since the portfolio changed a lot every 20 days and on these changes we should pay the transaction costs. We observe this strong turnover in particular during the crisis where the portfolio is often changed for more than $75 \%$. However, we also observe that the strong turnover is partially guaranteed from the preselection practice, since the "best" 100 preselected assets change every month.

\section{Conclusion}

In this work we have analysed mean-risk approaches to portfolio selection problem. We proposed two new dispersion measures taking into account the stochastic order due to additive shifts. In particular, we analysed and discussed the stochastic properties of these dispersion measures (colog and cologdsr). Finally, a comparison among different dispersion measures and a coherent risk measure $(\mathrm{CVaR})$ confirmed a very good performance of the newly suggested ones together to the conditional value at risk. From the comparison among the standard deviation, colog and cologdsr, we generally observed that the two new measures can generally provide a higher ex post final wealth. However, the conditional value at risk presents the highest ex-post final wealth even if for long periods presents lower performance than cologdsr whose performance appears comparable with the one of CVaR. Therefore this result appears promising for the new dispersion measures even considering that dispersion measures must be generally used in a different way by risk measures. In this context we have also proposed a methodology that is able to predict the periods of systemic risk. Moreover, we also analysed the diversification and the turnover of the different portfolio strategies that present similar behaviour with strong turnover and sufficient diversification. 


\section{References}

Artzner, P., Delbaen, F., Heath, J-M., Eber, D. (2000), "Coherent Measures of Risk." Mathematical Finance, Vol. 9, No. 3, pp. 203-228.

Bauerle, N., Müller, A. (2006), "Stochastic Orders and Risk Measures: Consistency and Bounds." Insurance: Mathematics and Economics, Vol. 38, No. 1, pp. 132-148.

Dybvig, P. (1988), “Distributional Analysis of Portfolio Choice." Journal of Business, Vol. 61, No. 3, pp. 369-393.

Hadar, J., Russel, W. (1969), "Rules of Ordering Uncertain Prospects." American Economic Review, Vol. 59, No. 1, pp. 25-34.

Hanoch, G., Levy, H. (1969), "The Efficiency Analysis of Choices Involving Risk." Review of Economic Studies, Vol. 36, No. 3, pp. 335-346.

Konno, H., Yamazaki, H. (1991), “Mean-absolute Deviation Portfolio Optimization Model and Its Application to Tokyo Stock Market." Management Science, Vol. 37, No. 5, pp. 519-531.

Kopa, M., Tichý, T. (2014), "Comparison of Mean-risk Efficient Portfolios in Asia-Pacific Capital Markets." Emerging Markets Finance and Trade, January-February 2014, Vol. 50, No. 1, pp. 226-240.

Levy, H. (1992), "Stochastic Dominance and Expected Utility: Survey and Analysis." Management Science, Vol. 38, No. 4, pp. 555-593.

Markowitz, H. (1959), Portfolio Selection; Efficient Diversification of Investment. New York: Wiley.

Ogryczak W., Ruszczyński, A. (1999), “From Stochastic Dominance to Mean-Risk Models: Semideviations as Risk Measures." European Journal of Operational Research, Vol. 116, No. 1, pp. 33-50.

Ogryczak, W., Ruszczynski, A. (2001), "On Consistency of Stochastic Dominance and Mean Semideviation Models." Mathematical Programming, Vol. 89, No. 2, pp. 217-232.

Ogryczak W., Ruszczyński, A. (2002), “Dual Stochastic Dominance and Related Mean-risk Models." SIAM Journal on Optimization, Vol. 13, No. 1, pp. 60-78.

Ortobelli S., Rachev S., Stoyanov S., Fabozzi F., Biglova A. (2005), “The Proper Use of the Risk Measures in the Portfolio Theory." International Journal of Theoretical and Applied Finance, Vol. 8, No. 8, pp. 1107-1133.

Ortobelli S., Rachev S., Shalit H., Fabozzi F. (2009), "Orderings and Probability Functionals Consistent with Preferences." Applied Mathematical Finance, Vol. 16, No. 1, pp. 81-102.

Ortobelli, S., Tichý, T. (2011), "On the Impact of Association Measures Within the Portfolio Dimensionality Reduction Problem." Central European Review of Economic Issues, Vol. 14, No. 4, pp. 257-264.

Peters O. (2011), "Optimal Leverage from non Ergodicity." Quantitative Finance, Vol. 11, No. 11, pp. 1593-1602.

Petronio, F., Lando, T., Biglova, A., Ortobelli, S. (2014), “Optimal Portfolio Performance with Exchange-traded Funds." Central European Review of Economic Issues, Vol. 17, No. 1, pp. 5-12.

Rachev, S., Mittnik, S. (2000), Stable Model in Finance. New York: Wiley. 
Rachev, S., Ortobelli, S., Stoyanov, S., Fabozzi, F., Biglova, A. (2008a), “Desirable Properties of an Ideal Risk Measure in Portfolio Theory." International Journal of Theoretical and Applied Finance, Vol. 11, No. 1, pp. 19-54.

Rockafellar, R. T., Uryasev, S., Zabarankin, M. (2006), "Generalized Deviations in Risk Analysis." Finance and Stochastics, Vol. 10, No. 1, pp. 51-74.

Rothschild, M., Stiglitz, J. (1970), "Increasing Risk: I. definition." Journal of Economic Theory, Vol. 2, pp. 225-243.

Shaked, M., Shanthikumar, G. (1993), Stochastic Orders and Their Applications. New York: Academic Press.

Speranza, M. G. (1993), "Linear Programming Models for Portfolio Optimization." Finance, Vol. 14, pp. 107-123.

Zenios, S. A., Kang, P. (1993), "Mean-absolute Deviation Portfolio Optimization for Mortgagebacked Securities." Annals of Operations Research, Vol. 45, pp. 433-450. 